\title{
The Cause and Eliminate Method of Self-excited Oscillation in Long Cable Amplifier Circuits
}

\author{
Can Wang ${ }^{1, a}$, Ming Huang ${ }^{1, b}$, Zhaomin Peng ${ }^{1, c}$, Jinbao Cui ${ }^{1, d}$ \\ ${ }^{1}$ North China University of Technology, Beijing, 100144, China
}

\begin{abstract}
SMA long cable distributed capacitance is easy to trigger broadband amplifier circuit's selfoscillation. In this study we design an amplifier circuit based on OPA691 chip, discuss the influence of long cable's distributed capacitance, then prompt a solution to add $50 \Omega$ compensation resistor between the output terminal and the load capacitance. According to the Tina simulation analysis, when $50 \Omega$ compensation resistor is added, bode diagrams show amplitude characteristic is smaller than $0 \mathrm{~dB}$ at $-180^{\circ}$ phase. The selfoscillation also disappeared in actual circuit measurement, which verify the rationality of this solution.
\end{abstract}

\section{INTRODUCTION}

Most of the operational amplifier circuit inevitably contains capacitive load components, such as distributed capacitance of the PCB conductor or coaxial cable, these objective existence will affect the operational amplifier characteristics, in the case of broadband it easily trigger instability work, or even self-oscillation phenomenon. This paper will explore the principle and the eliminate measures of broadband amplifier self-excited oscillation caused by SMA long cable distribution capacitor.

\section{PRINCIPLE OF SELF-EXCITED OSCILLATION}

Fig.1 shows a schematic diagram of a feedback amplifier. $\dot{A}$ is the amplification factor of basic amplifier, $\dot{F}$ is the feedback coefficient of the feedback network.

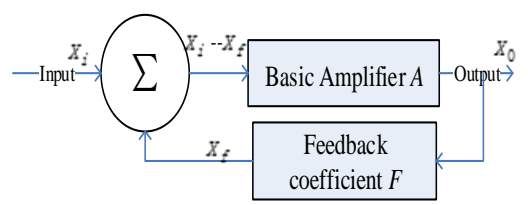

Figure1: Principle of self-excited oscillation ${ }^{[1]}$

From which can deduced the gain $\mathrm{A}_{f}$ of the amplifier circuit with negative feedback as follows:

$$
A_{f}=\frac{\dot{X}_{0}}{\dot{X}_{l}}=\frac{\dot{X_{0}}}{\dot{X}_{0} / \dot{A}+\dot{X}_{0} \dot{F}}=\frac{\dot{A}}{1+\dot{A} \dot{F}}
$$

The self-excited phenomenon must meet the selfoscillation conditions, feedback loop coefficient : $\dot{A} \dot{F}=-1$.

The conditions consist of two parts: a. Amplitude condition is: $|\dot{A} \dot{F}|=1$

b. Phase condition is:

$\angle \dot{A} \dot{F}=\varphi_{A}+\varphi_{F}=(2 \mathrm{n}+1) \pi \quad(\mathrm{n}=0,1,2 \cdots \cdots)$

Where $\varphi_{A}$ is the phase of the amplifier $\dot{A}, \varphi_{F}$ is the phase of the feedback network $\dot{F}, \varphi_{A}+\varphi_{F}$ is the phase difference between the feedback signal and the input signal. When actual circuit meets the phase condition and positive feedback, also satisfy $\varphi_{A}+\varphi_{F} \in\left[(2 n+1) \pi-\frac{\pi}{4}\right.$, $\left.(2 n+1) \pi+\frac{\pi}{4}\right](n=0,1,2 \cdots)$, the circuit is in anstable state, which may also produce self-oscillation ${ }^{[2]}$.

The physical meaning of the above description is: If normalized vector signals which enter the broadband amplifier at one moment into a signal unit, then the broadband amplifier will output the corresponding vector of $1 \times \dot{A}$ units; and then through the feedback network $\dot{F}$ back to the broadband amplifier, will returne corresponding vector of $1 \times \dot{A} \times \dot{F}$ units; if $1 \times \dot{A} \times \dot{F}=1$, it means that without external input signals, the feedback signal can maintained in the reciprocal transfer feedback path, keeping the same energy ${ }^{[3]}$. This means that the system can output equal amplitude oscillations without input -that is, self-excited oscillation.

\section{SMA LONG CABLE DISTRIBUTED CAPACITANCE'S IMPACT ON OPERATIONAL AMPLIFIER}

SMA coaxial cable's center coaxial conductor and outer shield metal can be equivalent to the distributed capacitance as shown in Fig.2.Refer to the manufacturer's information, we use SMA distributed capacitance about $96.1 \mathrm{pf} / \mathrm{m}$. So introduce the SMA cable equivalent of increasing the capacitive load of broadband amplifier

\footnotetext{
* Corresponding author: ${ }^{2} 2269718459 @$ qq.com, ${ }^{2}$ huangming@ncut.edu.cn, $362951939 @$ qq.com, d1195791948@qq.com
} 
circuit, and impact the stability of the entire amplifier system $^{[3]}$.

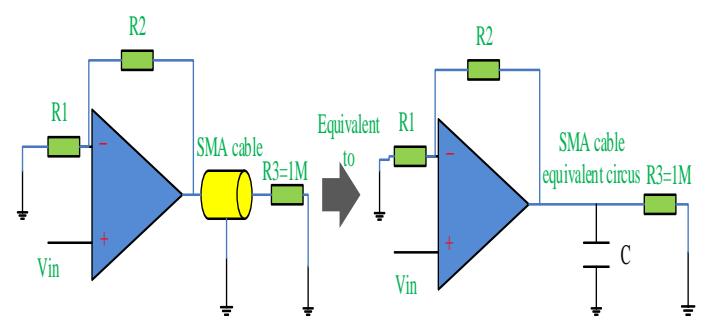

Figure2: Distributed capacitance equivalent model

In $1.5 \mathrm{~m}$ line simulation, the distribution of capacitance value is similar to $96.1 \mathrm{pf} / \mathrm{m} * 1.5 \mathrm{~m} \approx 150 \mathrm{pf}$; the broadband amplifier circuit using TI's OPA691 chip (set the Rf bandwidth to $260 \mathrm{MHz}$ or less) to build 8 times the magnification of the in-phase amplification circuit. OPA691 open-loop gain characteristics determine the amplitude characteristic of $\dot{A}: \dot{F}=\frac{R 1}{R 1+R 2}$. Refer the OPA691 manual comprehensive analysis Fig. 2 circuit $\dot{A} \dot{F}$ can be obtained in this ideal circuit should be stable, will not occur self-oscillation. This is also demonstrated by circuit simulation using the TINA simulation software provided by TI's official website, as it shows in Fig.3. However, after considering the influence of the distributed capacitance, the circuit is unstable and may occur selfoscillation in Fig.4.

\subsection{SIMULATION OF AMPLITUDE- FREQUENCY CHARACTERISTIC WITHOUT CAPACITIVE LOAD}

In the case of uncharged load, analogy the capacitance model of Fig.2 can simulated the circuit shown in Fig.3 left, the pote characteristic is shown in Fig. 3 right 。
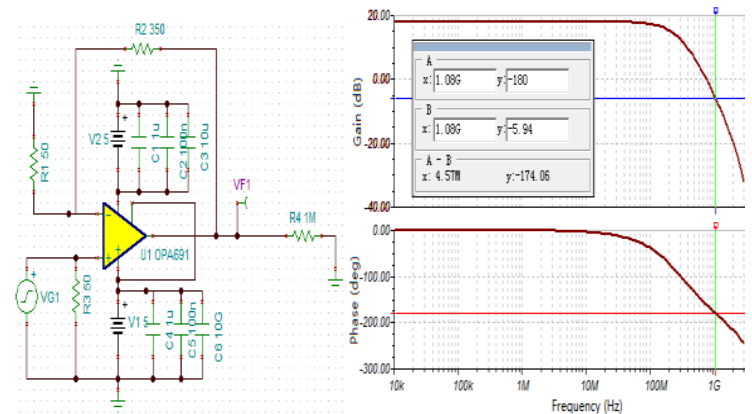

Figure3: Circuit and baot diagram without capacitive load

In the self-excited oscillation conditions, when $|\dot{A} \dot{F}|<1$, the output signal is continuously reduced, and will not occur self-oscillation. In the frequency of $-180^{\circ}$ phase, the amplitude characteristic is $-5.94 \mathrm{~dB}<0 \mathrm{~dB}$, the system is stable, will not occur self-oscillation. [Note: set Rf OPA691 bandwidth within $260 \mathrm{MHz}$, beyond this frequency will not occur self-oscillation, the specific derivation refer to OPA691 official website datasheet description $^{[4]}$. ]

\subsection{SIMULATION OF AMPLITUDE- FREQUENCY CHARACTERISTICS WITH CAPACITIVE LOAD}

The following circuit adds the equivalent capacitance to the SMA connected position ${ }^{[5]}$. The $2 \mathrm{~m}$ cable introduces about 200pf equivalent capacitance. The effect is shown in Fig.4.
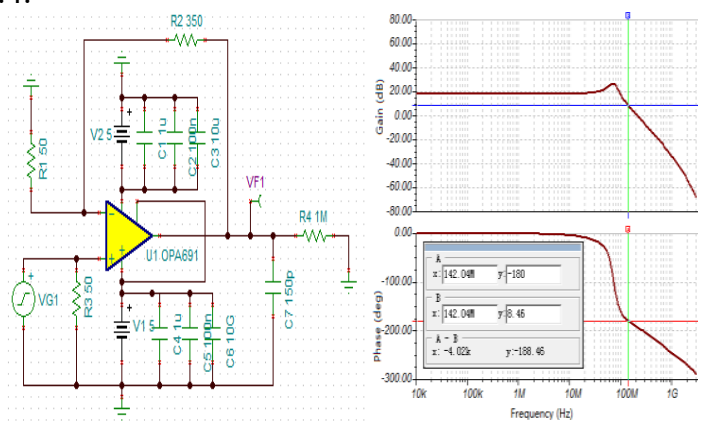

Figure4: Circuit and baot diagram with capacitive load

From the amplitude-frequency characteristic curve shows that the phase at $-180^{\circ}$, there is $142.04 \mathrm{MHz}$ frequency amplitude characteristics $8.46 \mathrm{~dB}>0 \mathrm{~dB}$, to meet the conditions of self-oscillation occurred. Therefore, in the case of $150 \mathrm{pF}$ capacitive load, instability will produce self-oscillation.

Through the above comparison simulation is obvious, capacitive load $C_{7}$ affect the system's amplitude-frequency characteristics, undermine the stability of the system.

\section{ANALYZE THE THEORY}

\subsection{CREATE A CIRCUIT MODEL}

To analyze the principle of the capacitance, the equivalent circuit of the amplifier circuit in Fig.4 must be analyzed. Because in fact the amplifier open-loop output resistance is not $0 \Omega$, so the first broadband amplifier OPA691 equivalent to for the flow control voltage source with a amplification factor is $\dot{A}$, the external output contains a resistance Ro, the official manual indicate $R o \approx 15 \Omega$, as shown in Figure 5.

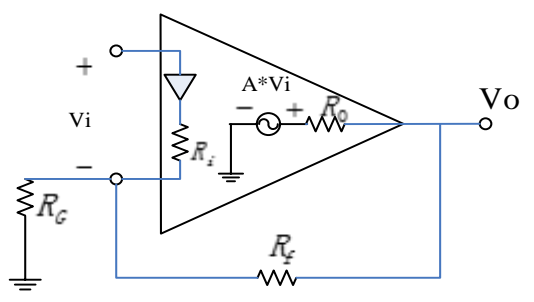

Figure5: Amplifier equivalent circuit

Open-loop simulate the OPA691 circuit , using $1 \mathrm{GH}$ inductance $L_{1}$ to rcuitdisconnect the AC loop, and through the $1 \mathrm{GF}$ introduce $C_{8}$ the $\mathrm{AC}$ ground attach to the inverting 
input, with $1 \mathrm{M} \Omega$ resistor load, then get the corresponding baud figure as shown in Fig.6.
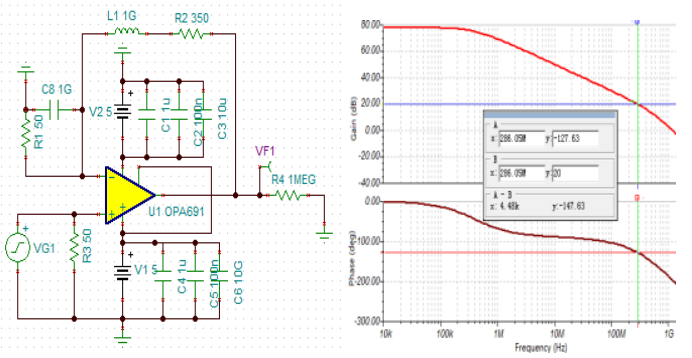

Figure6: Open-loop simulate circuit and the baot diagram

\subsection{CALCULATION}

In the actual simulation, we simulate the capacitive load and the no-capacitive load separately, find out the problem by contrast and solve the problem

\subsubsection{NO-CAPACITIVE LOAD ANALYSIS}

When the capacitive load $C_{7}$, the simulation shown in Figure 3. Theoretical calculation implies the feedback coefficient is a pure real number, phase change of $\angle \dot{A} \dot{F}$ only determined by the amplifier amplitude frequency characteristics $\dot{A}$.

$$
\dot{F}=50 /(350+50) \approx 1 / 8 \quad \angle \dot{F}=0
$$

$20 \log _{10}|\dot{A} \dot{F}| \approx 20 \log _{10}|\dot{A}|-20 \angle \dot{A} \dot{F}=\angle \dot{A}$

Add the $\beta=1 / \mathrm{F}=20 \mathrm{~dB}$ curve in the open-loop gain spectrum in Fig.6, the two-point intersection is $20 \log _{10}|\dot{A} \dot{F}|=0 \mathrm{~dB}$ frequency. The phase at this frequency point is $-127.63^{\circ}$ and does not occur self-oscillation.

\subsubsection{CAPACITIVE LOAD ANALYSIS}

With the load of capacitive $C_{7}$, the equivalent diagram shown in Fig.7 left side.

Theoretical calculation $\mathrm{Vo} \approx \mathrm{A} * \mathrm{Vi}^{*}\left[\frac{1}{\mathrm{j} \omega \mathrm{CL}} / / R_{L} / /\left(R_{f}+R_{G}\right)\right] /\left[R o+\frac{1}{\mathrm{j} \omega \mathrm{CL}} / / R_{L} / /\left(R_{f}+R_{G}\right)\right] ;$

When $R_{L} \gg R o, R_{f} \gg R o$, and $R_{f}>R_{G}$, the approximate calculation can ignored $R_{L}, R_{f}, R_{G}$ as shown in the right side of Fig. 7, $V o \approx \mathrm{A}^{*} \mathrm{Vi} *\left(\frac{1}{\mathrm{j} \omega \mathrm{CL}}\right) /\left(R o+\frac{1}{\mathrm{j} \omega \mathrm{CL}}\right)$; the feedback voltage $V_{f} \approx V_{o} * R_{G} /\left(R_{G}+R_{f}\right)=\mathrm{A} * V i * \frac{1}{\mathrm{j} \omega \mathrm{CL}} /\left(R_{o}\right.$ $\left.+\frac{1}{\mathrm{j} \omega \mathrm{CL}}\right) * R_{G} /\left(R_{G}+R_{f}\right)$. This is equivalent to adding a lowpass filter based on the 4.2.1 analysis, which means adding a pole.

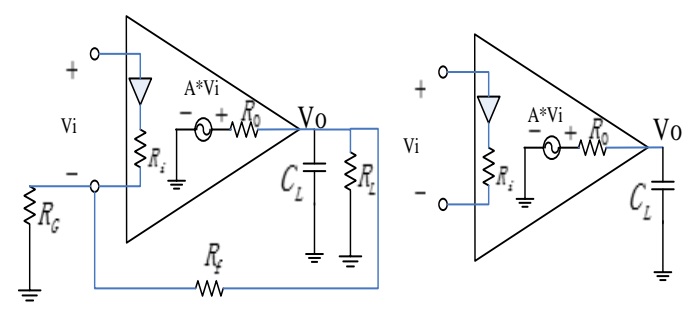

Figure7: Equivalent circuit and simplified diagram
According to Fig.3 and Fig.4, it can be observed that there is one more pole in Fig. 4 than Fig.3, and an inflection point occurs at a frequency of $70.9 \mathrm{MHz}$. The verification of this pole is:

$$
f_{\mathrm{p}}=\frac{1}{2 \pi C \cdot R_{0}}
$$

When $R o \approx 15 \Omega, C \approx 150 \mathrm{pF}$, calculate the pole frequency is about $71 \mathrm{MHz}$, basically same as the simulation results.

\section{SELF-EXCITED OSCILLATION ELIMINATION}

The reason for self-oscillation occurs after capacitive load added is that pole produced damage the stability of the circuit. So we need to introduce a zero point after the main pole position, thus suppressing the influence of the pole and maintaining the stability of the circuit ${ }^{[6]}$.

As equivalent circus shows on the left of Fig.8, resistor $R_{5}=50$ is placed outside the loop of the amplifier, then connected to the load $C_{7}$ capacitor.

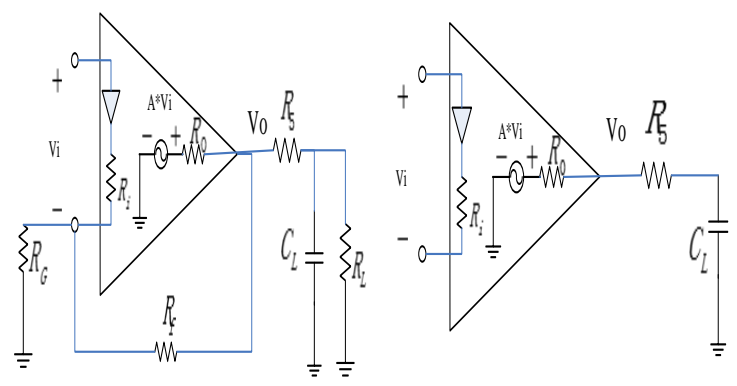

Figure8: Compensation circuit and simplified diagram

According to the calculate formula:

$\quad V o \approx A^{*} V i^{*}\left[\left(\frac{1}{\mathrm{j} \omega \mathrm{CL}} / / R_{L}+R_{5}\right) / /\left(R_{f}+R_{G}\right)\right] /\{R o$
$\left.+\left[\left(\frac{1}{\mathrm{j} \omega \mathrm{CL}} / / R_{L}+R_{5}\right) / /\left(R_{f}+R_{G}\right)\right]\right\} ;$
When $R_{L} \gg\left(R_{0}+R_{5}\right) 、 R_{f} \gg R_{G}$ 、且 $R_{f} \gg>\left(R_{0}+\right.$ When $R_{L} \gg\left(R_{0}+R_{5}\right) 、 R_{f} \gg R_{G}$ 、且 $R_{f}>>\left(R_{0}+\right.$
$\left.R_{5}\right)$, approximate calculations can ignore $R_{L}, R_{f}, R_{G}$, as shown in Fig.8 on the right.

$V o \approx A^{*} \mathrm{Vi}^{*}\left[\left(\frac{1}{\mathrm{j} \omega \mathrm{CL}}+R_{5}\right)\right] /\left[R o+\left(\frac{1}{\mathrm{j} \omega \mathrm{CL}}+R_{5}\right)\right] ;$

So, the feedback voltage is:

$V_{f} \approx V o^{*} R_{G} /\left(R_{G}+R_{f}\right)=A^{*} V i^{*}\left(\frac{1}{\mathrm{j} \omega \mathrm{CL}}+R_{5}\right) /\left(R o+\frac{1}{\mathrm{j} \omega \mathrm{CL}}+\right.$ $\left.R_{5}\right) * R_{G} /\left(R_{G}+R_{f}\right)$

This is equivalent to adding a zero point on the basis of 4.2.2 analysis.

$$
f_{z 1}=\frac{1}{2 \pi R_{5} C_{L}}
$$

After adding $R_{5}$, the position of $f_{p}$ produced by $R_{0}$ and $C_{L}$ changed, now it is determined by $R_{0}, R_{5}$ and $C_{7}$ :

$$
f_{p 2}=\frac{1}{2 \pi\left(R_{5}+R_{0}\right) C_{7}}=\frac{1}{2 \pi \times 65 \times 150 \times 10^{-12}} \approx 16.3 \mathrm{MHz}
$$

At the same time introduce a zero point $f_{z}$ after the first pole to suppress the impact of the pole on the system, which location determined by $R_{5}$ and $C_{7}$ :

$f_{z 1}=\frac{1}{2 \pi R_{5} C_{7}}=\frac{1}{2 \pi \times 50 \times 150 \times 10^{-12}} \approx 21.2 \mathrm{MHz}$

Simulation result shows in Fig.9: 

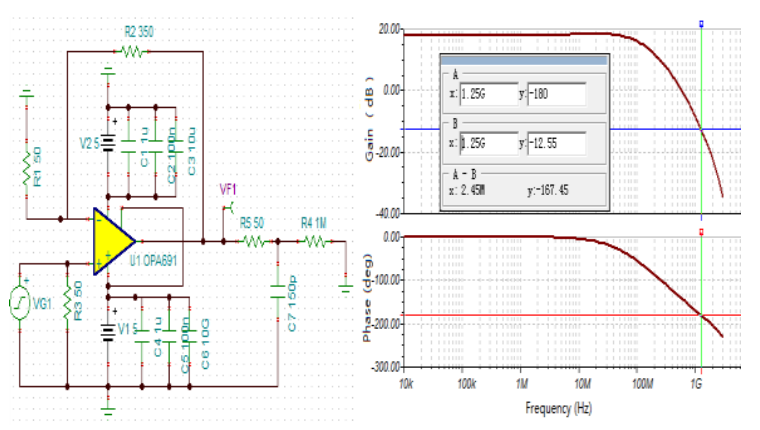

Figure9: Compensation circuit model

From the amplitude-frequency characteristic curve shows, there is $1.25 \mathrm{GHz}$ frequency amplitude characteristics $-12.55 \mathrm{~dB}<0 \mathrm{~dB}$ at $-180{ }^{\circ}$ phase, and set RF below $260 \mathrm{Mhz}$, there is no frequency instability and positive feedback phenomenon. It proves this method can successfully elimination the self-oscillation.

\section{CIRCUIT MEASUREMENT}

The test circuit shown in Fig.10, in the actual measurement, Vf2 grounded through $50 \Omega$ resistor, Vf1 through the SMA cable connected to the oscilloscope which input probe impedance $>1 \mathrm{M} \Omega$.
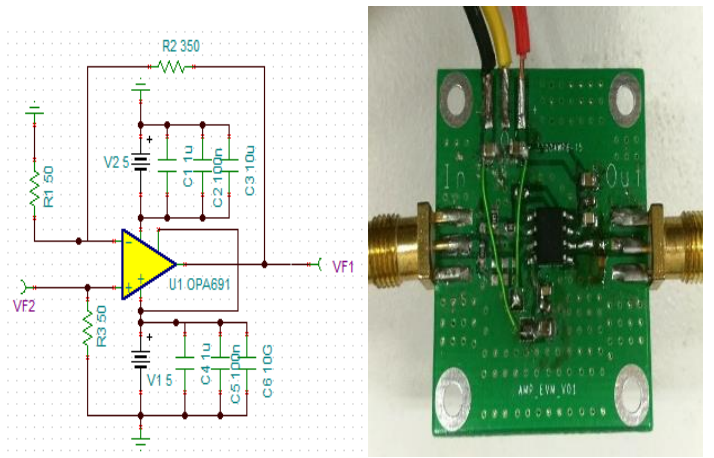

Figure10: Test circuit

Test equipment: Agilent DSO7054A oscilloscope; Test power supply: $\pm 5 \mathrm{~V}$ DC voltage source;

Test cable: ancient wave semi-flexible cable with different length of $2.2 \mathrm{~m}, 1.8 \mathrm{~m}, 1.5 \mathrm{~m}, 1.2 \mathrm{~m}, 80 \mathrm{~cm}, 60 \mathrm{~cm}$.

Results of testing:

Conntect different lengths of cable, the capacitance parameters will change, test results shown in Table 1.

TABLE 1: Measurement results

\begin{tabular}{|c|c|c|c|}
\hline $\begin{array}{c}\text { Length } \\
/ \mathbf{m}\end{array}$ & $\begin{array}{l}\text { Equivalent } \\
\text { capacitance } \\
\text { /pf }\end{array}$ & $\begin{array}{c}\text { Frequency } \\
/ \mathbf{M h z}\end{array}$ & $\begin{array}{c}\text { Self-oscillation } \\
\text { After introduce } \\
\mathbf{5 0} \text { resistor } \\
\text { (Yes/No) }\end{array}$ \\
\hline 2.2 & 211.4 & 64.5 & No \\
\hline 1.8 & 173.0 & 76.1 & No \\
\hline 1.5 & 144.15 & 90.4 & No \\
\hline 1.2 & 115.32 & 36.7 & No \\
\hline 0.8 & 76.9 & 50.5 & No \\
\hline 0.6 & 38.4 & 63.8 & No \\
\hline
\end{tabular}

According to the summary table, it can be clearly concluded that adding a capacitive load causes the amplifier system generate self-oscillating oscillation. By introducing a $50 \Omega$ series resistor between the output of the op amp and the load capacitor, the self-oscillating oscillation can be eliminated.

\section{SUMMARY}

This paper analyzes the self-oscillation of the broadband amplifier caused by SMA long cable distribution capacitance, explain and simulate the reason of the selfoscillation of the circuit with capacitive load. At the same time propose the method of solving this problem, that is, introducing a $50 \Omega$ series resistor between the output of the op amp and the load capacitor to eliminate the self-excited oscillation. The method proved feasibility in both theory and actual test in various lengths cable.

\section{REFERENCES}

1. C.Y.HUA and S.B.TONG, Analog electronic technology foundation,(Higher Education Press,BeiJing, 2006).

2. Texas Instruments, Texas Instruments High Performance Analog Devices Funny Application Guide,(2013),pp.70-89.

3. J.G.YANG, Hello Amplifier,(Analog devices support community,2014), https://ezchina.analog.com/community/amplifiers

4. Texas Instruments, OPA691 Datasheet, see http://www.ti.com/product/opa691.

5. S.P.SUN, D.SUN and F.ZHANG, "Design and Simulation of Virtual Self-oscillating Circuits", Journal of Electrical \& Electronic Education, 2016,38(05), pp.96-98+119.

6. W.S.SUN, T.Y.Tan and G.W.GONG, "Analysis of Operational Amplifiers Circuits with the negative feedback model", Electronic Technology, 2009,46(03), pp.87-88. 